\title{
Localization of Microfibers within Volumes Using Digital Holographic Video
}

\author{
${\text { Emmanouil Darakis }{ }^{d, a,{ }^{*}} \text {, Michel Kempkes }}^{c}$, Taslima Khanam ${ }^{a}$, Arvind Rajendran ${ }^{a}$, Vinay Kariwala ${ }^{a}$, \\ Thomas J. Naughton ${ }^{d, e}$, Marco Mazzotti ${ }^{c}$, and Anand K. Asundi ${ }^{b}$. \\ ${ }^{a}$ School of Chemical and Biomedical Engineering, Nanyang Technological University, Singapore, 637459; \\ ${ }^{b}$ School of Mechanical and Aerospace Engineering, Nanyang Technological University, Singapore, 639798; \\ ${ }^{c}$ Institute of Process Engineering, ETH Zurich, CH-8092 Zurich, Switzerland; \\ ${ }^{d}$ Department of Computer Science, National University of Ireland - Maynooth, Co Kildare, Ireland; \\ ${ }^{e}$ University of Oulu, RFMedia Laboratory, Oulu Southern Institute, Vierimaantie 5, 84100 Ylivieska, Finland. \\ *Corresponding author e-mail address:emmdarakis@ieee.org
}

\begin{abstract}
Carbon fibers are studied using digital holographic sequences. Fibers have distinct orientation in space. An algorithm to identify the orientation and the size of suspended fibers from the digitally recorded holographic sequences is presented.

(C)2009 Optical Society of America

OCIS codes: (090.1995) Digital holography; (100.6890) Three-dimensional image processing; (180.6900) Threedimensional microscopy; (350.4990) Particles
\end{abstract}

\section{Introduction}

Digital holography has been used in numerous metrology applications [1]. A particularly interesting application of digital holography is in particle analysis where relatively large volumes of samples can be studied with a single hologram recording, as opposed to photography based techniques where the depth of field imposed by the magnification optics limits the size of the studied volume [2].

Usually, spherical particles are used in these studies. As a result of the spherical particles' symmetry, such studies are limited to the measurement of the particles' diameter and location in space [2]. In this work carbon fibers are studied, for which, owing to their axis symmetry, 2 rotational degrees of freedom also need to be determined.

Sequences of digital holograms recorded from a fiber with a controllable orientation over time are analyzed. It is shown that with appropriate processing of the recorded holographic sequences, the orientation of the fiber within a volume and its size can be accurately determined.

\section{Localization of Fibers within Volumes}

For the recording of the digital holograms the in-line setup shown in Fig. 1 is used. A spherical diverging illumination beam is created by focusing a green laser $(\lambda=532 \mathrm{~nm})$ on a $1 \mu \mathrm{m}$ pinhole by a $60 \times$ microscope objective lens. The diverging beam illuminates the sample. Part of the beam is diffracted by the particles within the sample creating the object beam. Another part of the illumination beam passes through the sample undiffracted providing the reference beam. The object and the reference beam interfere and a camera is used to record their interference pattern.

The sample consists of a single carbon fiber (type T383, Toho Tenax Europe GmbH, Wuppertal, Germany) which is $1320 \mu \mathrm{m}$ long (measured with a microscope). The fiber is placed on a glass slide which is mounted on a device that can be manually rotated as shown in Fig. 1. The camera records digital holograms at a rate of 1 hologram per second while the tilt of the fiber along the optical axis is manually controlled. Fig. 2(a) shows one of the recorded holograms. 


\section{DTuB6.pdf}

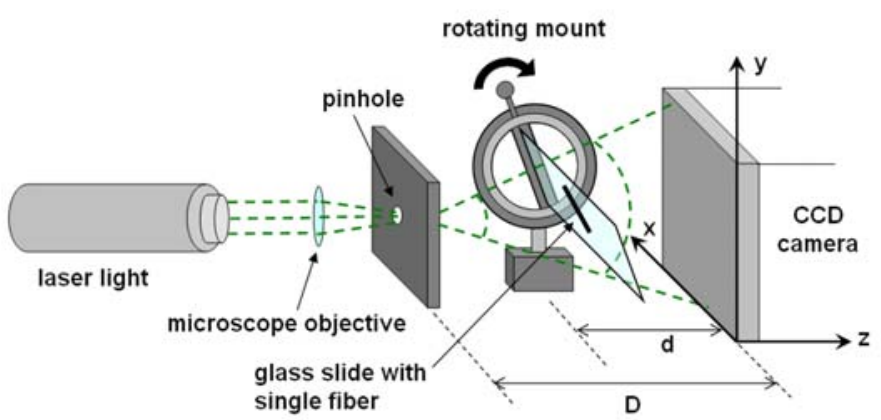

Fig. 1. Recording setup.



(a)

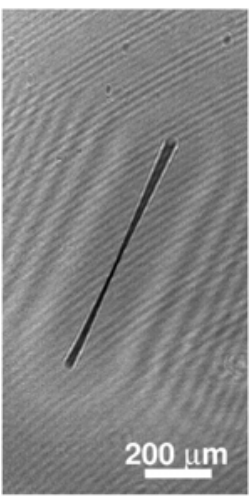

(b)



(c)



(d)

Fig. 2. Three dimensional profiling of a fiber tilted to $45^{\circ}$ along the optical axis: (a) recorded hologram, (b) the intensity of a reconstruction, (c) thresholded reconstruction, and (d) 3D point cloud with fitted line.

In order to determine the orientation of the fiber from the recorded holographic sequences the procedure described next is followed. Each hologram is reconstructed using the Fresnel - Kirchhoff diffraction formula [1] at several reconstruction depths covering the volume that needs to be studied. Fig. 2(b) shows one of the reconstructions of the hologram shown in Fig. 2(a). The fiber shown in this hologram is tilted along the optical axis to $45^{\circ}$ and hence, as it can be seen, only its middle section is focused at this particular reconstruction distance.

Following this, the intensity of the reconstructed hologram is thresholded with a low threshold value $t$ (typically $5 \%$ of the maximum intensity value). Pixel values with intensity higher than $t$ are set to ' 0 ' and the rest to ' 1 '. Fig. 2(c) shows the thresholded reconstruction shown in Fig. 2(b). As it can be seen from Fig. 2(c), in this way the section of the fiber that is well focused at this particular reconstruction distance can be determined. This procedure is repeated for all the reconstruction depths. The resulting thresholded reconstructions are stacked together to form a 3D matrix. Everything within this 3D matrix is zero apart from a point cloud that corresponds to the fiber.

The volume studied might contain more than one fiber, so the algorithm has to identify each of them individually. To this end, based on their 3D connectivity, the points of each cloud are grouped together and labeled. The resulting point clouds have the size and the orientation of the studied fibers and hence these quantities can be measured accurately from the respective point clouds. For this, Principal Component Analysis (PCA) is used to fit a straight line to the data points corresponding to each of the identified fibers [3].

In practice, due to noise, parts of the fibers might not appear focused at any depth, causing single fibers to appear as 2 or more separate ones. In order to identify such cases, following line fitting, collinear fibers with ends close to each other are merged together to restore the single fiber. Fig. 2(d) shows the 3D point cloud of the fiber shown in the hologram and the line fitted to it. As it can be seen, the length and the orientation of the line within the volume correspond to the length and the orientation of the fiber. 


\section{DTuB6.pdf}

\section{Results and discussion}

By repeating the described fiber identification procedure for all the frames of a holographic sequence, the movement of the fibers within the volume can be tracked over time. Fig. 3 shows the identified position of a rotating fiber at different time instances. The method can be used to track the orientation of fibers over time and hence it has the potential to be used as an extension of Holographic Particle Image Velocimetry (HPIV) [4], where the orientation of the fibers over time can provide additional flow information.

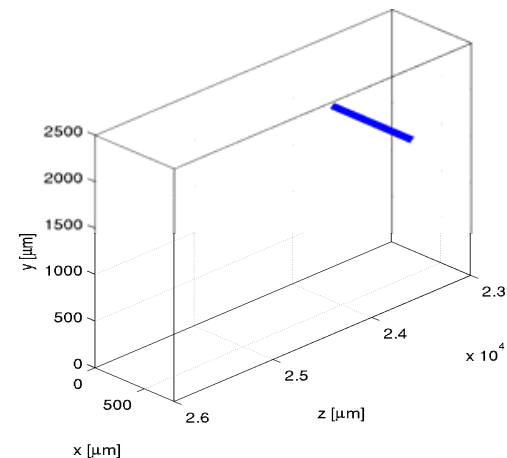

(a)



(b)

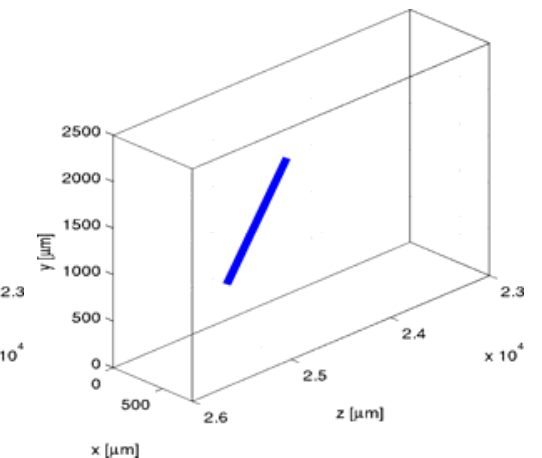

(c)

Fig. 3. Identified position and orientation of a fiber at three different moments in time.

Previously proposed particle size measurement methods using digital holography, such as the one reported in [5], measure the size of the projection of the particles on the plane of the camera. Although this approach works well for spherical particles, measuring fiber lengths based on their projection would lead to an error that is proportional to the tilt of each fiber. For example, as it can be seen in Fig. 2(b), the length of this projection is $\sim 650 \mu \mathrm{m}$ resulting in an error of about $50 \%$, given that the real length of the fiber is $1320 \mu \mathrm{m}$. The method proposed here takes into account the orientation of the fiber and hence measures its length correctly. As a result, it can be used for accurate measurement of fibers in suspension [6].

To conclude, a method to identify the position and the orientation of carbon fibers within volumes by analyzing holographic sequences recorded by digital cameras has been presented. The method can be used for accurate size measurement of fibers in suspension as well as to track their 3D motion and orientation.

Funding by the Irish Research Council for Science, Engineering and Technology under the National Development Plan through grant no. PD/2007/11 and the Office of Research, Nanyang Technological University through grants no. RG25/07 and M58120012 are acknowledged. The authors would also like to thank Qu Weijuan for assisting with the recording setup.

[1] U. Schnars and W. Jueptner, Digital holography: digital hologram recording, numerical reconstruction, and related techniques (Springer, 2005).

[2] J. Sheng, E. Malkiel, and J. Katz, "Digital holographic microscope for measuring three-dimensional particle distributions and motions," Apl. Opt. 45, 3893-3901 (2006).

[3] S. Wold, K. Esbensen, and P. Geladi, “Principal component analysis,” Chemom. Intell. Lab. Syst. 2, 37-52 (1987).

[4] K. D. Hinsch, “Holographic particle image velocimetry,” Meas. Sci. Technol. 13, R61-R72 (2002).

[5] W. Xu, M. H. Jericho, I. A. Meinertzhagen, and H. J. Kreuzer, “Digital in-line holography of microspheres,” Appl. Opt. 41, 5367-5375 (2002).

[6] M. Kempkes, E. Darakis, T. Khanam, A. Rajendran, V. Kariwala, M. Mazzotti, T. J. Naughton, and A. K. Asundi, “Three dimensional digital holographic profiling of micro-fibers," Op. Ex. submitted for publication (2008). 